\title{
Lessons of the month: Massive gastrointestinal bleeding in a young woman with idiopathic thrombocytopenic purpura (ITP)
}

\author{
Authors: Ksheetij Kothari, ${ }^{A}$ Mallikarjun Patil, ${ }^{B}$ Renuka Malipatel ${ }^{C}$ and Harshad Devarbhavi ${ }^{D}$
}

\begin{abstract}
Cytomegalovirus (CMV) is a ubiquitous pathogen, belongs to the herpes virus family and can infect the gastrointestinal (GI) system. The disease is usually noted in immunocompromised patients such as solid organ transplant recipients on immunosuppressive drugs, patients with malignancy receiving chemotherapy, patients with AIDS, patients on steroids for autoimmune disorders, and is rarely seen in immunocompetent individuals. In the GI system, CMV most commonly involves the colon, followed by oesophagus, stomach and, rarely, the small intestine. The GI manifestation of CMV infection is usually anorexia, diarrhoea, and blood in stools, abdominal pain and fever. Very rarely, CMV infection may present with a massive GI bleed. We report a case of 36-year-old pregnant woman with idiopathic thrombocytopenic purpura (ITP) who presented with massive GI bleeding following delivery, attributed to isolated CMV enteritis.
\end{abstract}

KEYWORDS: cytomegalovirus, enteritis, massive gastrointestinal bleeding

DOI: $10.7861 /$ clinmed.2020-0803

\section{Case presentation}

A 36-year-old woman, 34 weeks pregnant, presented with bleeding from her vagina. She was diagnosed with idiopathic thrombocytopenic purpura (ITP) and was taking steroids (prednisolone $10 \mathrm{mg} /$ day) for the previous 5 months. On examination, she was haemodynamically stable and had evidence of fetal distress, so she was then taken for emergency lower segment caesarean section. Intraoperatively, she was found to have placenta accreta and required a hysterectomy to control the bleeding.

Authors: ${ }^{\text {A }}$ senior resident, St John's Medical College Hospital, Bangalore, India; ${ }^{B}$ professor, St John's Medical College Hospital, Bangalore, India; ' assistant professor, St John's Medical College Hospital, Bangalore, India; ${ }^{D}$ professor and head of department of gastroenterology, St John's Medical College Hospital, Bangalore, India
On postoperative day 16 , she developed fresh blood from her rectum. There was no history of abdominal pain, diarrhoea, fever nor intake of non-steroidal anti-inflammatory drugs (NSAIDs). There was no history suggestive of epistaxis, gum bleeding, haematuria or skin rash. Her medication history included prednisolone $10 \mathrm{mg} /$ day and pantoprazole. On clinical examination, she was afebrile, blood pressure of $110 / 70 \mathrm{mmHg}$, pulse rate of 80 beats per minute and respiratory rate of 18 breaths per minute. She had mucosal pallor and no petechiae on the skin. Her abdomen was soft and non-tender with normal bowel sounds. A digital rectal examination revealed fresh blood. Laboratory parameters showed haemoglobin $(\mathrm{Hb})$ of $8 \mathrm{~g} / \mathrm{dL}$ (drop by $2 \mathrm{~g}$ ), platelet count $86 \times 10^{9} / \mathrm{L}$ and total leukocyte count of $7.8 \times 10^{9} / \mathrm{L}$. Coagulation profile and liver function tests were normal. An upper gastrointestinal (GI) endoscopy appeared normal until the third part of the duodenum and colonoscopy examination showed fresh blood in the ileum with normal-appearing colonic mucosa suggestive of source of bleeding proximal to ileum and distal to duodenum. Postprocedure, she developed hypotension with passage of massive amount of fresh blood. She was transferred to the intensive care unit and resuscitated with intravenous fluid therapy and packed cell transfusion. Her blood investigations revealed low $\mathrm{Hb}(4 \mathrm{~g} / \mathrm{dL})$ and platelet count was $90 \times 10^{9} / \mathrm{L}$. Computed tomography (CT) angiography did not reveal a source of bleeding. She continued to have GI bleeding with haemodynamic instability despite resuscitative measures, hence subjected to emergency laparotomy with intraoperative enteroscopy. Enteroscopy showed a large amount of fresh blood in the distal jejunum and proximal ileum with multiple, discrete, actively bleeding ulcers (Fig 1). The diseased segment was resected $(80 \mathrm{~cm})$ and end-to-end anastomosis was done.

Gross examination of resected small bowel revealed large punched-out ulcers (Fig 2). On histopathological examination, characteristic intranuclear owl's eye inclusion bodies were seen in endothelial and stromal cells suggesting cytomegalovirus (CMV) infection (Fig 3). The serum CMV polymerase chain reaction test revealed viraemia $(2,000$ copies $/ \mathrm{mL})$. She was treated with intravenous ganciclovir 300 mg every 12 hours and an additional 3 units of packed cell and platelet transfusion. Although bleeding was controlled, her general condition deteriorated due the development of multi-organ failure. She died 5 days after surgery despite antiviral therapy and other supportive care. 


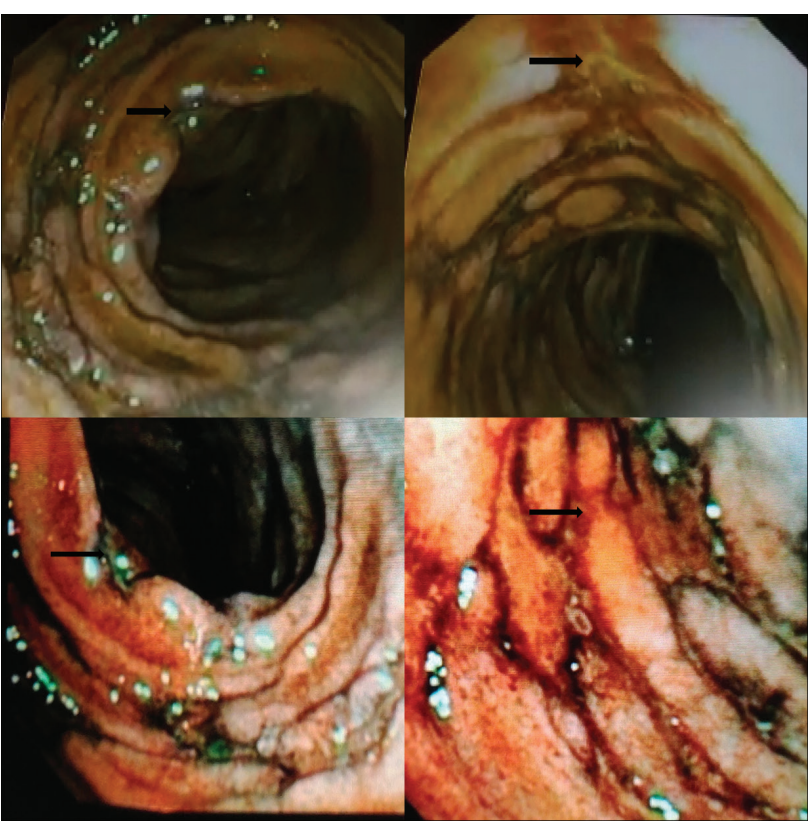

Fig 1. Enteroscopy showing multiple, discrete ulcers in jejunum (arrows).

\section{Discussion}

Our patient had obscure overt gastrointestinal bleeding (bleeding from unknown source after standard endoscopy, colonoscopy and radiological imaging). ${ }^{1}$ The common causes in young individuals are small bowel ulcers due to inflammatory bowel disease, NSAIDs intake and small bowel tumours (such as gastrointestinal tumours (GIST), lymphoma, neuroendocrinal tumours and carcinoma), Meckel's diverticulum, Dieulafoy's lesion and telangiectasia. ${ }^{2}$ After the negative standard endoscopy and colonoscopy examination, further investigations to ascertain the source of bleeding include $\mathrm{CT}$ angiography to rule out a structural cause. If the $\mathrm{CT}$ is normal, further evaluation includes capsule endoscopy and push

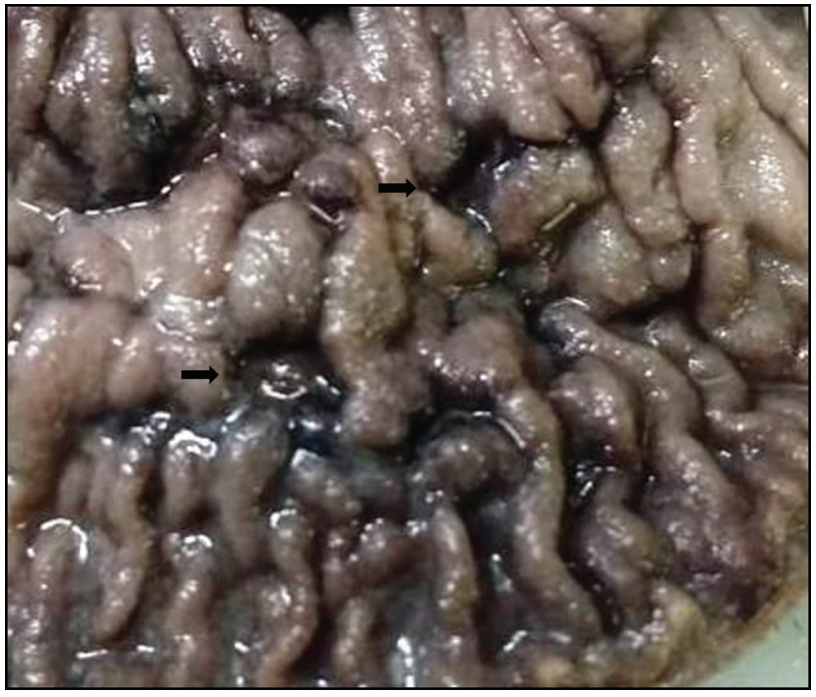

Fig 2. Resected part of small bowel showing punched out ulcers (arrows).

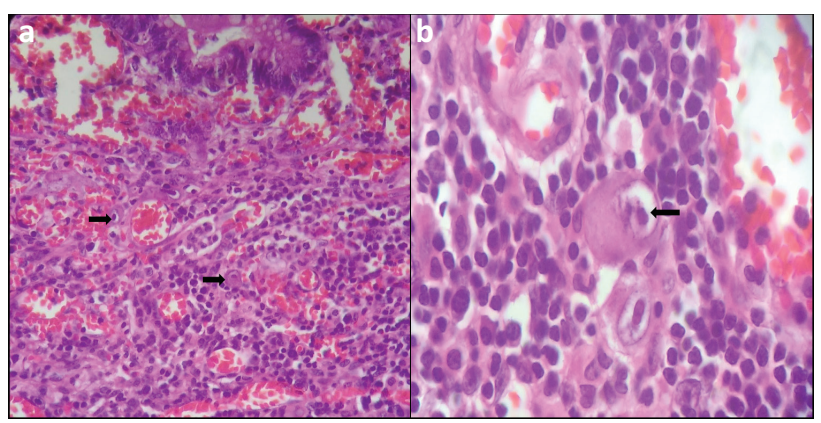

Fig 3. Haematoxylin and eosin stain showing ulceration, granulation tissue and enlarged stromal cells with characteristic intranuclear owl's eye inclusion bodies. a) Magnification $\times 400$. b) Magnification $\times 1,000$.

enteroscopy in haemodynamically stable individuals. Rarely in haemodynamically unstable patients (as with our patient), when CT doesn't reveal the cause and the requirement of surgical resection is imminent, intraoperative enteroscopy is required to ascertain the source of bleeding and surgical treatment can be done at the same time. ${ }^{3}$

Primary CMV infection is usually acquired in the first 20 years of life, which is either asymptomatic or may have self-limiting mononucleosis (such as symptoms in immunocompetent individuals). ${ }^{4}$ Following primary infection, CMV remains dormant in host tissue in harmony with host immunity and gets reactivated with suppressed immune response. The CMV infection of the gastrointestinal tract is usually due to reactivation of the dormant CMV due to immunosuppression and rarely due to superinfection of the diseased GI tract, like in inflammatory bowel disease. CMV can infect any part of GI system from oesophagus to rectum, but commonly involves colon (55\%), oesophagus and stomach (40\%) and, rarely, the small intestine $(4.3 \%){ }^{5}$

CMV infects vascular endothelial cells and surface epithelium of the small bowel and causes sub-epithelial haemorrhages, erosions, and superficial and deep ulcers which manifest with persistent diarrhoea, weight loss, obscure GI bleeding, intestinal obstruction, perforation and, rarely, massive GI bleeding. ${ }^{6}$ The pathogenesis of massive GI bleeding is due to vasculitis, vascular erosion and deep ischaemic ulcers. There are few case reports of massive GI bleeds due to CMV enteritis in literature. ${ }^{7-10}$ In most of the published cases, patients were immunocompromised. Diagnosis of CMV enteritis is difficult due to non-specific presentation and requirement of enteroscopy for histopathological diagnosis. Presence of CMV inclusion bodies is considered to be diagnostic. In our patient, diagnosis was done on basis of histopathological examination of a surgically resected specimen which showed presence of CMV inclusion bodies. Ganciclovir therapy is the treatment of choice. For massive GI bleeds, surgical resection is to be considered if embolisation therapy is not feasible/fails.

\section{Conclusion}

CMV enteritis can rarely present as massive GI bleed. It needs to be suspected as one of the causes of GI bleed in immunecompromised patients and needs to be confirmed by enteroscopy. Early initiation of anti-viral therapy may avert the need for surgery. Delayed diagnosis increases morbidity and mortality. 


\section{References}

1 Raju GS, Gerson L, Das A, Lewis B. American Gastroenterological Association (AGA) Institute technical review on obscure gastrointestinal bleeding. Gastroenterology 2007;133:1697-717.

2 Gerson LB, Fidler JL, Cave DR et al. ACG clinical guideline: Diagnosis and management of small bowel bleeding. Am J Gastroenterol 2015;110:1265-87.

3 Zaman A, Sheppard B, Katon RM. Total peroral intraoperative enteroscopy for obscure GI bleeding using a dedicated push enteroscope: diagnostic yield and patient outcome. Gastrointest Endosc 1999;50:506-10.

4 Godgame RW. Gastrointestinal cytomegalovirus disease. Ann Intern Med 1993;119:924-35.

5 Chamberlain RS, Atkins S, Saini N, White JC. Ileal perforation caused by cytomegalovirus infection in a critically ill adult. J Clin Gastroenterol 2000;30:432-5.

6 Iwasaki T. Alimentary tract lesions in cytomegalovirus infection. Acta Pathol Jpn 1987;37:549-65.
7 Keates J, Lagahee S, Crilley P, Haber M, Kowalski T. CMV enteritis causing segmental ischemia and massive intestinal hemorrhage. Gastrointest Endosc 2001;53:355-9.

8 Varma V, Perera MT, Olliff S et al. Cytomegalovirus ileitis causing massive gastrointestinal haemorrhage in a patient following hepatic resection. Trop Gastroenterol 2011;32:145-7.

9 Morunglav M, Theate I, Bertin G, Hantson P. CMV enteritis causing massive intestinal hemorrhage in an elderly patient. Case Rep Med 2010;2010:385795.

10 Choi SW, Chung JP, Song YK et al. Lower gastrointestinal bleeding due to cytomegalovirus ileal ulcers in an immunocompetent man. Yonsei Med J 2001;42:147-51.

Address for correspondence: Prof Mallikarjun Patil, Department of Gastroenterology, St John's Medical College Hospital, Sarjapur Road, Bangalore 34, Karnataka, India. Email:drmalli_arjun@yahoo.co.in 